\title{
Gene gun-mediated DNA vaccination induces antitumor immunity against human papillomavirus type 16 E7-expressing murine tumor metastases in the liver and lungs
}

\author{
C-H Chen ${ }^{1,2}, \mathrm{H} \mathrm{Ji}{ }^{3}, \mathrm{KW} \mathrm{Suh}{ }^{4,5}, \mathrm{MA} \mathrm{Choti}^{4}, \mathrm{DM}$ Pardoll $^{1}$ and T-C Wu ${ }^{1,3,6,7}$ \\ Departments of ${ }^{1}$ Oncology, ${ }^{3}$ Pathology, ${ }^{4}$ Surgery, ${ }^{6}$ Obstetrics and Gynecology, and ${ }^{7}$ Molecular Microbiology and Immunology, The \\ Johns Hopkins Medical Institutions, Baltimore, Maryland, USA; ${ }^{2}$ Department of Internal Medicine, National Taiwan University \\ Hospital, National Taiwan University, Taipei, Taiwan; and ${ }^{5}$ Department of Surgery, School of Medicine, Ajou University, South \\ Korea
}

DNA vaccination has emerged as an attractive approach for tumor immunotherapy. The aim of this study was to evaluate the potency of DNA vaccines in preventing and treating the liver and lung metastases of a human papillomavirus-16 (HPV-16) E7-expressing murine tumor (TC1). We used the gene gun method to vaccinate $C 57 B L / 6$ mice intradermally with DNA vaccines containing the HPV16 E7 gene, the E7 gene linked to the sorting signals of the Iysosome-associated membrane protein-1 (Sig/E7/ LAMP-1), or the 'empty' plasmid vector. The in vivo antitumor immunity was analyzed in both tumor prevention and tumor regression experiments. In addition, cytotoxic T lymphocyte (CTL) assays, enzyme-linked immunospot assay and enzyme-linked immunoabsorbent assay were used to assess the E7-specific $T$ cell-mediated and humoral immunity. Mice vaccinated with Sig/E7/LAMP-1 DNA generated the strongest E7-specific CTL activities, the highest numbers of E7-specific CD8 $8^{+}$cell precursors and the highest titers of E7-specific antibodies. While both E7 DNA and Sig/E7/LAMP-1 DNA generated potent antitumor immunity in the liver and lung metastases models, the Sig/E7/LAMP1 DNA was more potent under stringent conditions. DNA vaccination with E7-expressing plasmids was effective in controlling liver and lung metastases of an E7-expressing murine tumor. Our data suggest that antigen-specific DNA vaccination can potentially be applied to control liver and lung metastases of tumors with defined tumor-specific antigens.

Keywords: DNA vaccine; metastasis; human papillomaviruses; LAMP-1; antitumor; E7

\section{Introduction}

Metastasis is a common cause of death in many cancer patients. Conventional therapies, such as surgery, chemotherapy and radiotherapy, have limited success in controlling cancer metastases. The ideal cancer therapy should act systemically in addition to its local antitumor effect. In this regard, immunotherapy is especially attractive because it has the potency to eradicate systemic tumors in multiple sites in the body and the specificity to discriminate between neoplastic and non-neoplastic cells. $^{1}$

Tumor-specific antigens, when efficiently presented by antigen presenting cells (APCs) to both $\mathrm{CD}^{+}$cytotoxic T lymphocytes (CTLs) and $\mathrm{CD}^{+}$helper T cells, are capable of inducing potent $\mathrm{T}$ cell-mediated immunity - the most crucial component of antitumor immunity. There are several additional advantages of using an antigen-specific cancer vaccine. It is less likely to generate nonspecific

Correspondence: T-C Wu, Department of Pathology, The Johns Hopkins Hospital, 600 North Wolfe Street, Baltimore, Maryland 21205, USA Received 28 June 1999; accepted 31 August 1999 autoimmunity, more flexible in deciding the amount of antigen administered and the methods of antigen presentation to the immune system, more reproducible from patient to patient and has the potential to correlate clinical outcome to a specific immune response. ${ }^{2}$ Therefore, antigen-specific cancer immunotherapy represents a desirable approach for controlling tumors.

In the past few years, several reports have shown that antigen-specific cancer vaccines, in the form of cell-based vaccines, protein/peptide-based vaccines and viral vector-based vaccines, are effective for the treatment of metastases. ${ }^{1}$ However, production of the autologous tumor cell-based vaccines is labor intensive and technically challenging. ${ }^{3}$ The application of peptide-based vaccines is limited by major histocompatibility complex (MHC) restriction and the necessity of defining the CTL epitopes. The disadvantages of viral vector-based vaccines include unnecessary immune responses to vectors and other potential virus-associated toxicity. In contrast, naked plasmid DNA is safe, low immunogenically and can be repeatedly administered. DNA vaccines can be easily prepared on a large scale with high purity and are highly stable relative to proteins and other biologic polymers (for review, see Refs 4-6). 

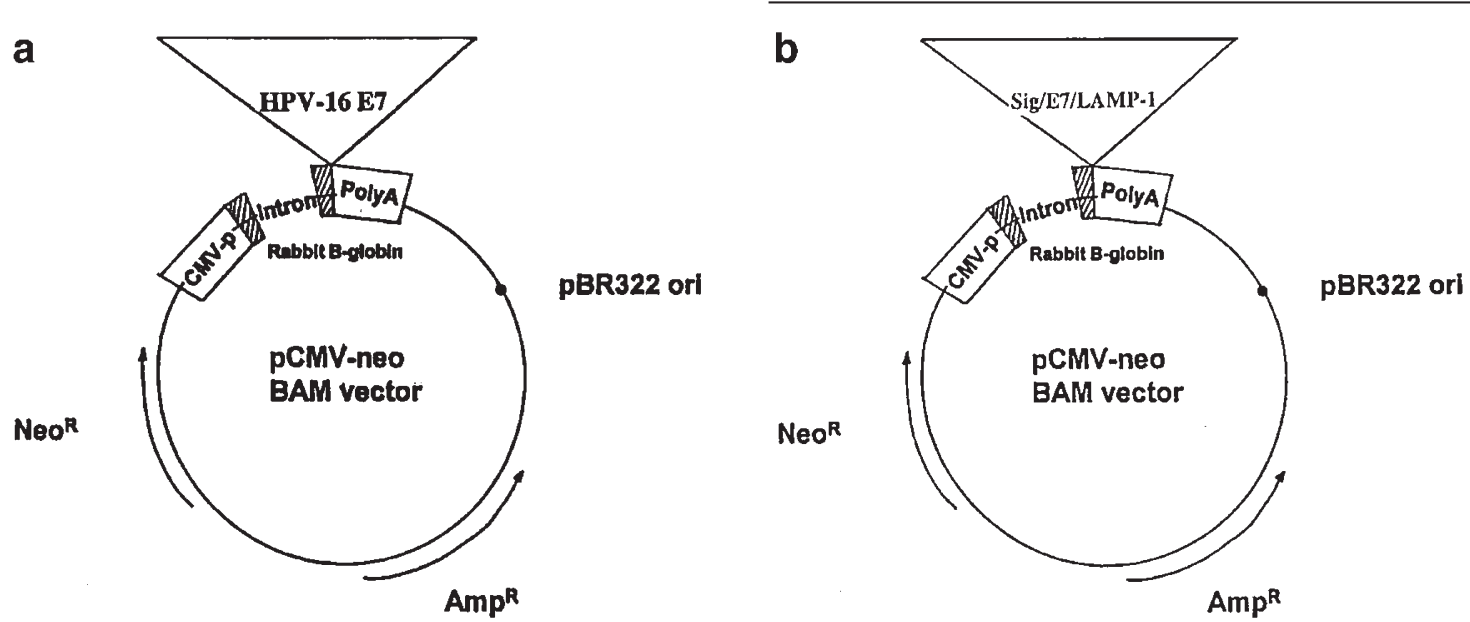

Figure 1 Schematic diagram of wild-type E7 and Sig/E7/LAMP-1 DNA constructs. The wild-type HPV-16 E7 gene and the chimeric Sig/E7/LAMP-1 gene were cloned into the BamHI cloning sites of the $\mathrm{PCMVneoBam} \mathrm{expression} \mathrm{vector} \mathrm{downstream} \mathrm{of} \mathrm{the} \mathrm{cytomegalovirus} \mathrm{promoter} \mathrm{as} \mathrm{described} \mathrm{in}$ Materials and methods.

We chose human papilliomavirus-16 (HPV-16) E7 as a model antigen for vaccine development because HPVs, particularly HPV-16, are associated with most cervical cancers. The HPV oncogenic proteins, E6 and E7, are important in the induction and maintenance of cellular transformation and are co-expressed in most HPV-containing cervical cancers. Therefore, vaccines or immunotherapies targeting E7 and/or E6 proteins may provide an opportunity to prevent and treat HPV-associated cervical malignancies.

We have previously linked the sorting signals of the lysosome-associated membrane protein-1 (LAMP-1) to the HPV-16 E7 antigen, creating a chimera, Sig/E7/LAMP-1 (see Figure 1). We found that mice vaccinated with Sig/E7/LAMP-1-containing recombinant vaccinia virus can generate strong antitumor immunity against HPV-16 E7-expressing tumors in subcutaneous, ${ }^{7}$ lung metastasis ${ }^{8}$ and hepatic metastasis models (Chen et $a l$, unpublished data). However, immune responses to the vaccinia vaccine vector can limit the effectiveness of revaccination with vaccinia virus. ${ }^{9}$ The use of naked DNA vaccines can circument this problem.

We used liver and lung metastasis models in the current study because besides regional lymph nodes, the liver and lungs are the most common sites of distant metastases of cervical cancers. ${ }^{10}$ In addition, when developing clinical applications for the treatment of common solid malignancies, demonstrating efficacy of therapy within visceral sites such as the liver or lung is important. ${ }^{11}$ Therefore, the aim of this study was to test whether DNA vaccines encoding HPV-16 E7 could induce protective immunity against HPV-16 E7-expressing murine tumor metastases in the liver or lungs.

\section{Results}

Vaccination with Sig/E7/LAMP-1 DNA generates potent E7-specific CD8 ${ }^{+} T$ cell-mediated immune response

$\mathrm{CD}^{+} \mathrm{T}$ lymphocytes are one of the most crucial components of the antitumor effect. ${ }^{12}$ CTL assay and enzyme linked immunospot (ELISPOT) assays were used to determine the E7-specific T cell-mediated immunity generated by DNA vaccine. For the standard chromium

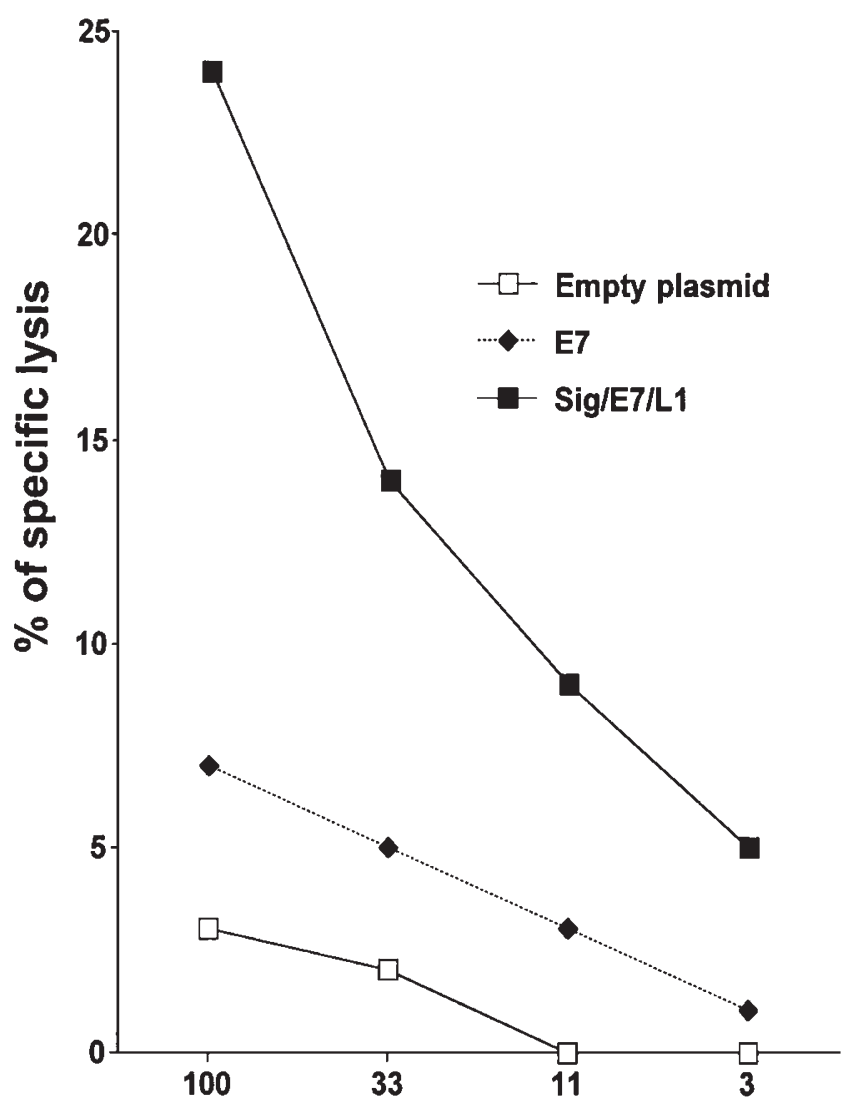

E:T ratio

Figure 2 CTL response in C57BL/6 mice immunized with various recombinant DNA vaccines. C57BL/6 mice were immunized with empty plasmid, E7 DNA or Sig/E7/LAMP-1 DNA via gene gun. Splenocytes were harvested 2 weeks after the last DNA vaccination and stimulated in vitro with E7-specific $H-2 D^{b}$ CTL epitope and interleukin-2 for 6 days. A standard $4 \mathrm{~h}$ chromium release assay was performed against MC57G $\left(\mathrm{H}-2^{b}\right)$ cells pulsed with E7 CTL peptide. Target cell-specific lysis was determined as described in Materials and methods. Results shown here are from one representative experiment of two performed. 


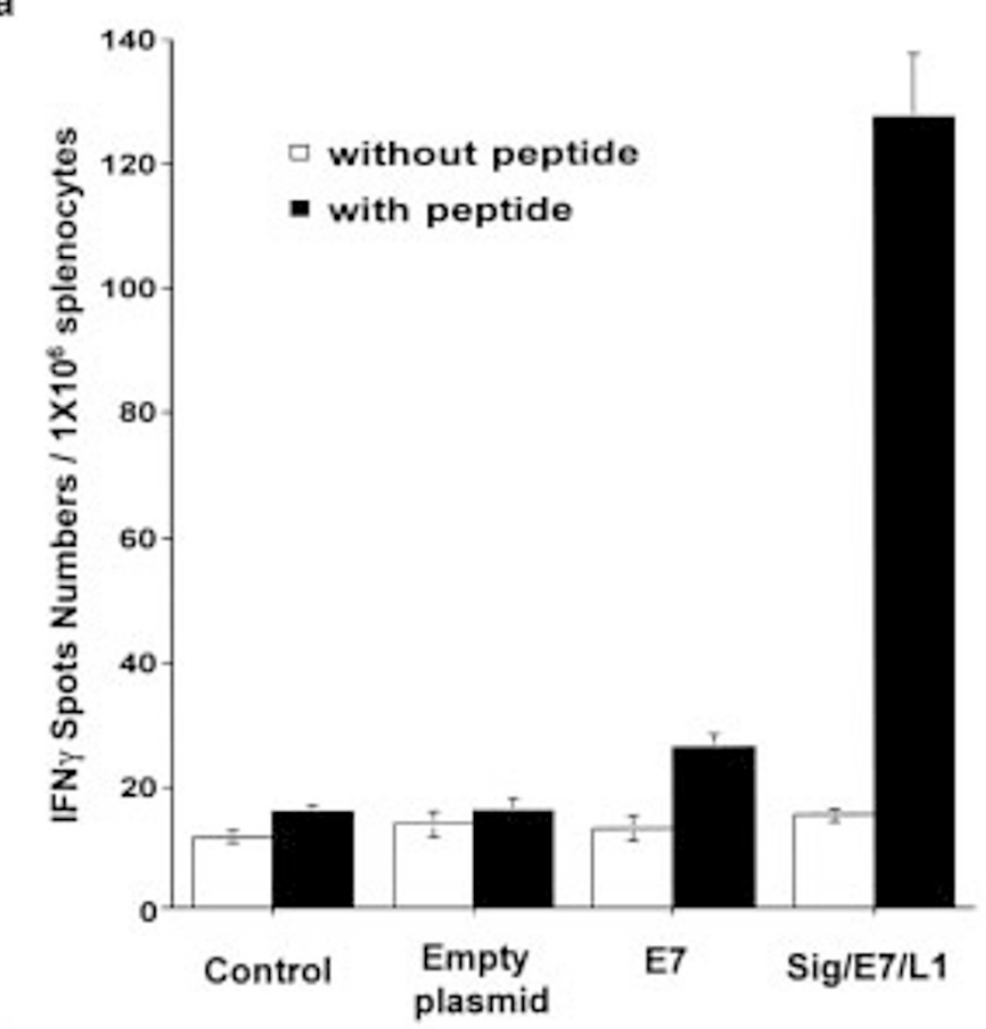

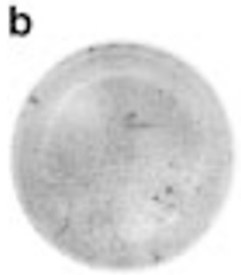

Control

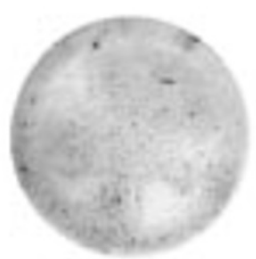

Empty

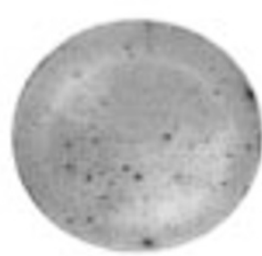

E7

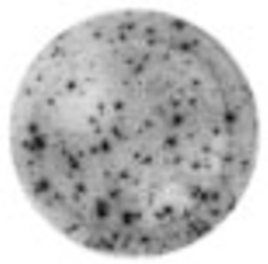

Sig/E7/L1

Figure 3 E7-specific CD8 ${ }^{+} T$ cell precursors in C57BL/6 mice immunized with various recombinant DNA vaccines using ELISPOT assays. C57BL/6 mice were immunized with empty plasmid, E7 DNA or Sig/E7/LAMP-1 DNA via gene gun. Splenocytes were harvested 2 weeks after the last DNA vaccination. The number of IFN- $\gamma$ producing E7-specific $C D 8^{+} T$ cell precursors was determined using the ELISPOT assay (see text for the detailed method). (a) The spot numbers were the mean of triplicates \pm s.e. in each vaccinated group. (b) Representative ELISPOT pictures in each vaccinated group. Results shown here are from one representative experiment of two performed.

release cytotoxicity assay, mice vaccinated with Sig/E7/LAMP-1 DNA generated the highest E7-specific CTL activity, while mice vaccinated with E7 DNA generated only slightly higher E7-specific CTL activity than the mice vaccinated with the empty plasmid (Figure 2). The ELISPOT assay is a sensitive function asaay for IFN- $\gamma$ production by individual cell and can be applied to quantify antigen-specific CD8 ${ }^{+} \mathrm{T}$ cell precursors. ${ }^{13}$ We used the ELISPOT assay to determine the precursor frequencies of E7-specific $\mathrm{CD}^{+} \mathrm{T}$ cells in each vaccination group. Around 120 E7-specific CD8 ${ }^{+} \mathrm{T}$ cells were detected per $10^{6}$ spenocytes derived from the Sig/E7/LAMP-1 DNAvaccinated mice, but only $10-20 / 10^{6}$ in E7 DNA-vaccinated mice. We did not detect E7-specific IFN- $\gamma$ secreting $\mathrm{CD}^{+} \mathrm{T}$ cells in control of empty plasmid-vaccinated mice (Figure 3). Our results from both CTL assay and ELISPOT assay indicated that Sig/E7/LASMP-1 DNA-vaccinated mice generated the highest $\mathrm{CD}^{+} \mathrm{T}$ cell-mediated immunity.

Vaccination with Sig/E7/LAMP-1 DNA generates the highest $T$ helper-type I cytokine responses

To determine the cytokine profiles generated by E7 DNA and Sig/E7/LAMP-1 DNA, splenocytes from vaccinated mice were stimulated in vitro with E7 protein for $72 \mathrm{~h}$. The supernatants were harvested and assayed for the presence of IFN- $\gamma$ and IL-4. As shown in Figure 4, the highest IFN- $\gamma$ level was found in the mice vaccinated with Sig/E7/LAMP-1 DNA. Vaccination with E7 DNA generated the second highest IFN- $\gamma$ release. IL-4 could not be detected in the supernatant of any vaccinated group. The detection limit was $5 \mathrm{pg} / \mathrm{ml}$ as determined from a linear standard curve. These results suggested 


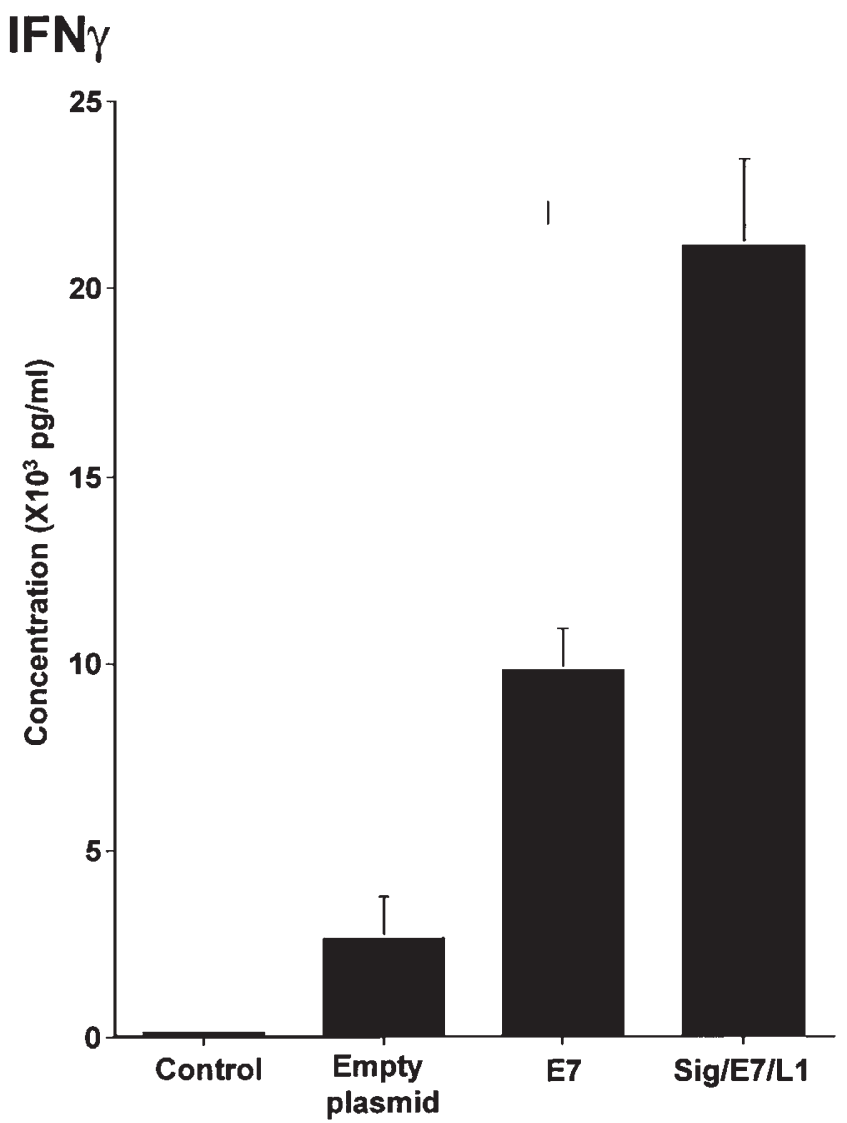

Figure 4 Cytokine secretion profiles of splenocytes in C57BL/6 mice immunized with various recombinant DNA vaccines. C57BL/6 mice were immunized with empty plasmid, E7 DNA or Sig/E7/LAMP-1 DNA via gene gun. Splenocytes $\left(4 \times 10^{6}\right)$ were harvested 2 weeks after the last DNA vaccination and were cultured with $10 \mu \mathrm{g} / \mathrm{ml}$ E7 protein in a 24-well tissue culture plate for $72 \mathrm{~h}$. The supernatants were harvested and assayed for specific cytokines using ELISA kit (Endogen). Results shown here are from one representative experiment of two performed.

that vaccination with Sig/E7/LAMP-1 DNA or E7 DNA vaccine generated $\mathrm{T}$ helper-type I cytokine profiles.

\section{Vaccination with Sig/E7/LAMP-1 DNA generates the highest E7-specific antibody titers}

The anti-HPV-16 E7 antibodies in the sera of the vaccinated mice were determined by a direct enzyme-linked immunoabsorbent assay (ELISA) at 1,2 and 4 weeks after the last vaccination. The anti-HPV-16 E7 antibodies could be detected in the sera of Sig/E7/LAMP-1 DNA-vaccinated mice at both 2 and 4 weeks after the last vaccination. No anti-E7 antibodies were detected in the sera of mice vaccinated with E7 DNA or the empty plasmid (Figure 5).

\section{Vaccination with both Sig/E7/LAMP-1 DNA and wild- type E7 DNA protects mice against TC-1 tumor metastases in the liver and lungs}

To determine whether vaccination with E7-expressing DNA constructs protects mice against E7-expressing tumor metastases in the liver and lungs, mice were vaccinated with $2 \mu \mathrm{g}$ naked DNA per mouse via gene gun, and boosted with the same dose 1 week later. Mice were then challenged with $1 \times 10^{4} \mathrm{TC}-1$ per mouse $(5 \times$ minimal

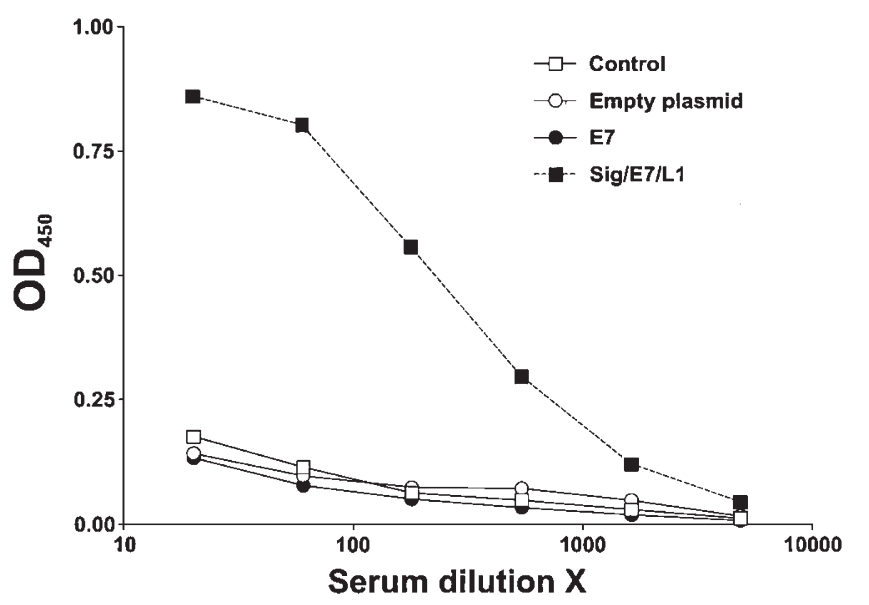

Figure 5 E7-specific antibody response in C57BL/6 mice immunized with various recombinant DNA vaccines. C57BL/5 mice were immunized with empty plasmid, E7 DNA or Sig/E7/LAMP-1 DNA via gene gun. Serum samples were obtained from mice 2 weeks after the last vaccination. A direct ELISA assay was performed. Results shown here are from one representative experiment of two performed.

tumor dose) in the liver (liver metastasis model) or $5 \times 10^{4}$ TC-1 per mouse $(10 \times$ minimal tumor dose) via intravenous tail vein injection (lung metastasis model) 1 week after the last vaccination. The mice were killed 30 days following tumor challenge.

For the liver metastases model, we found that all of the mice that received either E7 DNA or Sig/E7/LAMP1 DNA vaccination remained tumor-free in the liver. In contrast, all of the mice vaccinated with the empty plasmid and all of the naive mice developed hepatic tumors (Figure 6).

For the lung metastasis model, we counted pulmonary nodules to quantify tumor metastases in the lung. Mice vaccinated with either E7 DNA or Sig/E7/LAMP-1 DNA had the lowest mean number of pulmonary nodules (Figure 7). There was no statistical difference between E7 DNA and Sig/E7/LAMP-1 DNA. In our previous study, TC-1 tumors were detected only in the lungs after intravenous tail vein injection. Other solid organs including the brain, spleen, liver and kidneys remained free of tumors. Representative gross photographs of the lung tumors are shown in Figure 8.

\section{Vaccination with both Sig/E7/LAMP-1 DNA and wild- type E7 DNA eradicates established E7-expressing tumors in the liver and lungs}

To determine the therapeutic potential of E7-expressing DNA constructs in treating TC-1 tumor metastases in the liver and lungs, mice were first challenged with $1 \times 10^{4}$ TC-1 per mouse in the liver (liver metastases model) or $5 \times 10^{4}$ TC- 1 per mouse via intravenous tail vein injection (lung metastasis model). The mice were then vaccinated with $2 \mu \mathrm{g}$ naked DNA via gene gun 7 days later and boosted with the same dose 1 week later. The mice were killed 30 days after tumor challenge.

For the liver metastases model, we found that $80 \%$ of the mice vaccinated with E7 DNA or Sig/E7/LAMP-1 DNA remained tumor-free. In constrast, all naive mice and mice vaccinated with the empty plasmid developed hepatic tumors (Figure 9). 


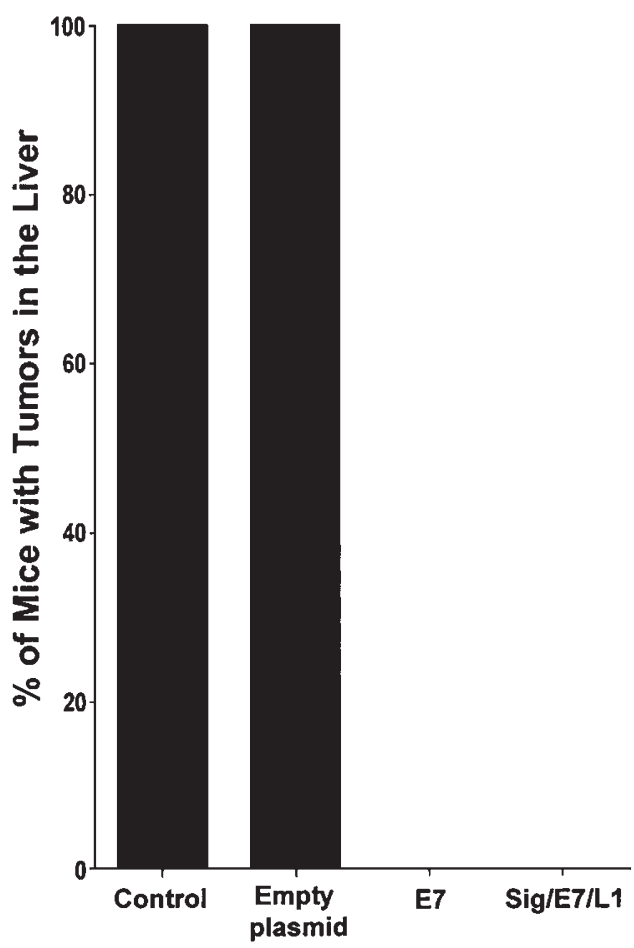

Figure 6 E7-expressing DNA vaccines protect mice against the growth of TC-1 tumors in the liver. C57BL/6 mice were immunized with empty plasmid, E7 DNA or Sig/E7/LAMP-1 DNA via gene gun. One week after the second vaccination, mice (five per group) were challenged with TC-1 tumor cells at a dose of $1 \times 10^{4}$ cells per mouse. Mice were monitored twice a week and were killed at day 30 after tumor challenge to check for the presence of liver tumors.

For the lung metastasis model, mice vaccinated with either E7 DNA or Sig/E7/LAMP-1 DNA had the lowest mean pulmonary nodule numbers (Figure 10). There was no statistical difference between E7 DNA and Sig/E7/LAMP-1 DNA.

These results showed that both E7 DNA and Sig/E7/LAMP-1 DNA could generate potent antitumor immunity in the liver and lung metastasis models of TC-1 tumor cells. However, there was no statisically significant difference in the antitumor effects between E7 DNA and Sig/E7/LAMP-1 DNA.

\section{Sig/E7/LAMP-1 DNA is a more potent vaccine than wild-type E7 DNA}

Both the humoral and cellular responses indicates that Sig/E7/LAMP-1 DNA may be a more potent DNA vaccine than E7 DNA. However, we cannot demonstrate the potency difference between Sig/E7/LAMP-1 DNA and E7 DNA in the in vivo antitumor effect by using the above vaccination and tumor doses. To test the difference between the antitumor potency generated by E7 DNA and that generated by Sig/E7/LAMP-1 DNA, we repeated the tumor protection and treatment experiments in the lung metastasis model under more stringent conditions. We decreased the DNA vaccine dose to $2 \mu \mathrm{g}$ per mouse (ie priming without further booster) and increased the tumor dose to $1 \times 10^{5} \mathrm{TC}-1$ per mouse. The mice were killed 21 days after tumor challenge. In the tumor protection experiment mice vaccinated with Sig/E7/LAMP-1 DNA had lower mean pulmonary nodule numbers com-

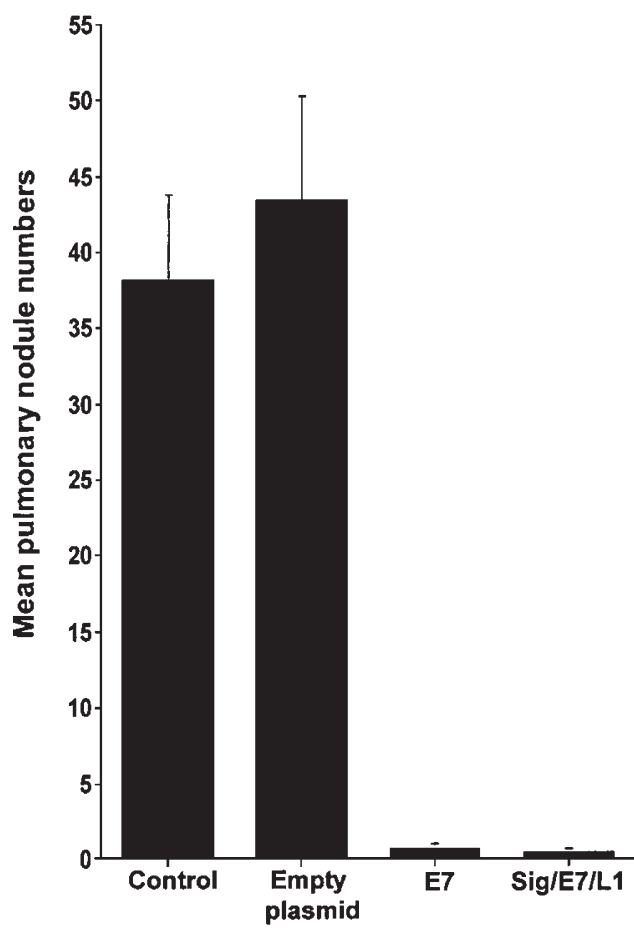

Figure 7 E7-expressing DNA vaccines protect mice against the growth of TC-1 tumors in the lungs. C57BL/6 mice were immunized with empty plasmid, E7 DNA or Sig/E7/LAMP-1 DNA via gene gun. One week after the second vaccination, mice (10 per group) were challenged with TC-1 tumor cells via intravenous tail vein injection at a dose of $5 \times 10^{4}$ cells per mouse. Mice were monitored twice a week and were killed at day 30 after tumor challenge. The mean pulmonary nodule numbers $( \pm$ s.e.) shown here are from one representative experiment of three performed.

pared with wild-type E7 DNA vaccination (Figure 11). In the tumor treatment experiments, there was no statistically significant difference in the mean pulmonary nodule numbers among Sig/E7/LAMP-1 DNA, E7 DNAvaccinated mice and the control mice (data not shown). These results indicated that under more stringent conditions, Sig/E7/LAMP-1 DNA vaccination generated a better antitumor effect than wild-type E7 DNA.

\section{Discussion}

In this study we demonstrated that gene gun-mediated DNA vaccination with Sig/E7/LAMP-1 DNA generated the strongest E7-specific humoral and cellular immunity. Both Sig/E7/LAMP-1 DNA and E7 DNA could generate significant preventive and therapeutic effects in HPV-16 E7-expressing murine tumors metastasis in the liver or lungs. Furthermore, the Sig/E7/LAMP-1 DNA demonstrated a stronger antitumor effect than E7 DNA under more stringent conditions (priming without further booster). Our results demonstrated the therapeutic efficacy of DNA immunization for the treatment of cancer metastases. Although DNA vaccination with intramuscular inoculation ${ }^{14-22}$ or intradermal injection via gene gun $^{23-25}$ have been shown to generate antitumor immunity for tumors inoculated subcutaneously, there are few reports of using DNA vaccines to treat cancer metastases. ${ }^{24}$ Our study represents a good example of using 


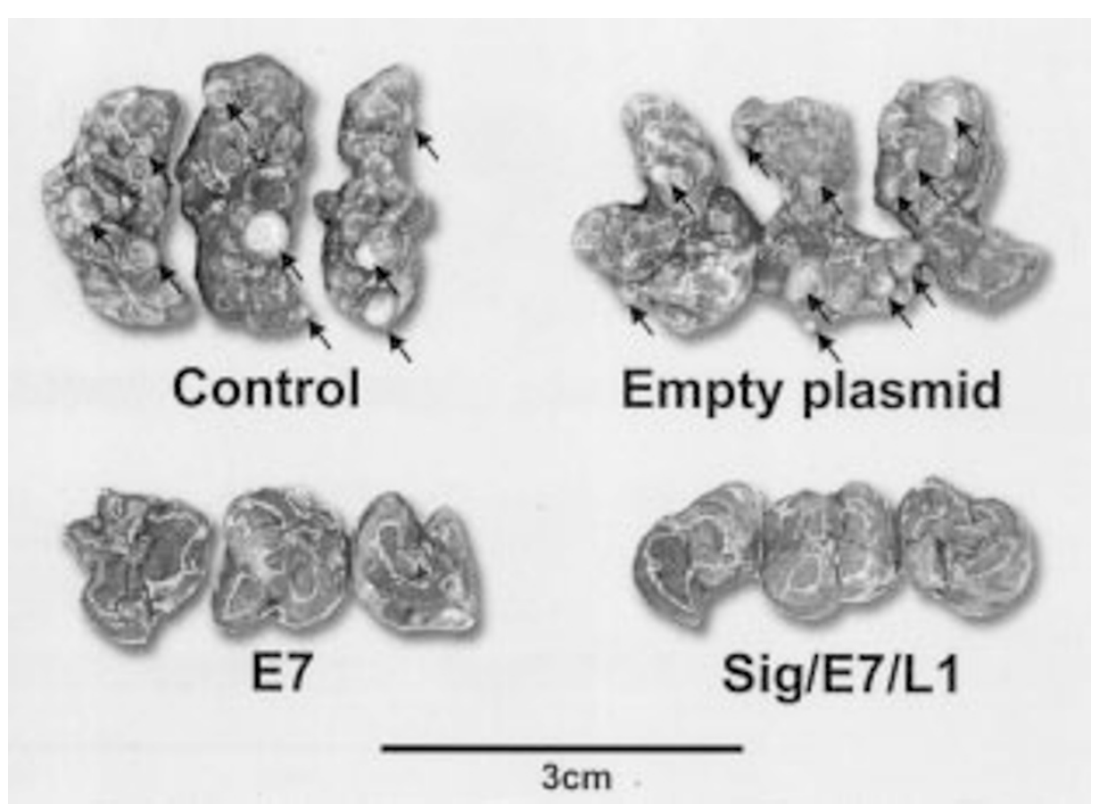

Figure 8 Representative gross pictures of the lung tumors in each vaccinated group. C57BL/6 mice were immunized with empty plasmid, E7 DNA or Sig/E7/LAMP-1 DNA via gene gun and were then intravenously injected with TC-1 tumor at $5 \times 10^{4}$ cells per mouse 7 days later. Mice were killed 30 days after tumor challenge. There are multiple grossly visible lung tumors in the control mice and mice vaccinated with empty plasmids (arrows). The rest of the lung tumors in each vaccinated group cannot be seen at the magnification in this figure.

antigen-specific DNA vaccine for the control of cancer metastases.

DNA vaccination is an attractive approach to generate antigen-specific vaccines because it induces cellular as well as humoral immunity with high efficacy. ${ }^{26}$ Even though the existing viral vectors are efficient in delivering DNA to targeted cells, DNA vaccines possess several advantages over these viral vectors. For example, immune recognition following adenoviral vector or vaccinia delivery inhibits repeat vaccination with the same delivery vector, while retroviral vectors are potentially toxic due to helper virus replication and insertional mutagenesis. In contrast, naked DNA vaccines have the clear advantages of purity, simplicity of preparation and stability. DNA-based vaccines can be prepared inexpensively and rapidly on a large scale. Furthermore, the CpG motif in plasmid DNA may cause simultaneous maturation of immature dendritic cells (DCs) and activation of mature $\mathrm{DCs}^{27}$ and thus can preferentially induce the $\mathrm{T}$ helper 1 response, ${ }^{28}$ which is important for the antitumor effect.

The exact mechanisms of DNA vaccines remain controversial in spite of the demonstrated antitumor immunity. Several studies have shown that bone marrow-derived APCs are the most important cells for presenting the antigen to the immune system in gene gun-mediated DNA vaccination. ${ }^{29,30}$ Following the gene gun delivery, the gene-transfected DCs travel to local lymph nodes, where the antigen-loaded DCs directly activate the naive $\mathrm{T}$ cells. ${ }^{29,31,32}$ This antigen presentation is probably not due to cross-presentation by untransfected DC of antigen released from or associated with transfected epidermal cells. ${ }^{31}$

As epidermal DCs are the professional APCs in gene gun-mediated DNA vacination, several points need to be addressed about the superiority of the Sig/E7/LAMP-1
DNA over the E7 DNA. Several pieces of indirect evidence imply that this superiority may be related to the ehnanced MHC-II presentation in DCs and the subsequent $\mathrm{CD}^{+} \mathrm{T}$ cell activation via the LAMP-1 targeting strategy. The enhanced MHC-II presentation by LAMP1 has been demonstrated previously in the HPV-16 E7 antigen $^{33}$ and human immunodeficiency virus gp160 antigen models ${ }^{34}$ using vaccinia virus as vectors. That Sig/E7/LAMP-1 DNA generated much higher E7-specific antibody titer than E7 DNA also indicated the help of $\mathrm{CD}^{+}$cells. Furthermore, intracellular cytokine staining showed that Sig/E7/LAMP-1 DNA generated higher numbers of IFN- $\gamma$-positive $\mathrm{CD}^{+} \mathrm{T}$ cells ( $\mathrm{Ji}$ and Wang, personal communication). Alternative explanations for the enhanced potency of Sig/E7/LAMP-1 DNA include protein expression in the chimeric constructs and the enhanced access of antigens to the endoplasmic reticulum via the signal peptide..$^{35,36}$

Although the superiority of Sig/E7/LAMP-1 DNA over E7 DNA in the antitumor effect was demonstrated, it was relatively difficult to see this 'narrow' window. Interestingly, it is easier to demonstrate that Sig/E7/LAMP-1 is more potent than E7 using vaccinia virus as vectors in both immunological assays and antitumor effects. ${ }^{7,33}$ This may imply that the mechanisms of the antitumor effects generated by the same constructs in the form of vaccinia vectors and naked DNA are different. The favored mechanism of gene gun-mediated DNA vaccination is the direct priming of naive $T$ cells by genetransfected DCs. ${ }^{31,37}$ In contrast, E7-expressing recombinant vaccinia virus may use the alternative $\mathrm{MHC}-1$ pathway (cross-priming) for the initiation of CTL responses ${ }^{38}$ in addition to the direct priming route. The enhanced CD4 presentation by LAMP-1 can help the induction of $\mathrm{CD}^{+}$CTL responses by cross-priming. ${ }^{39}$ Thus, $\mathrm{CD}^{+}$cellmediated help may be more important for cross-priming 


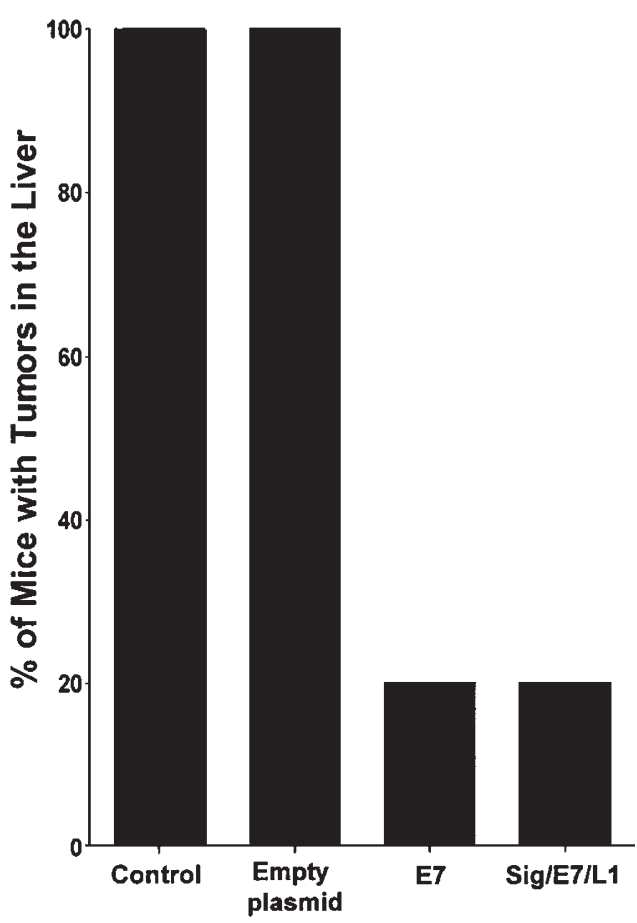

Figure 9 E7-expressing DNA vaccines eradicate previously established TC-1 tumors in the liver. C57BL/6 mice (five per group) were injected intrahepatically with TC-1 tumor at $1 \times 10^{4}$ cells per mouse and were immunized with empty plasmid, E7 DNA or Sig/E7/LAMP-1 DNA via gene gun 7 days later. Mice were killed 30 days after tumor challenge to check for the presence of liver tumors.

than for direct priming. This hypothesis may partially explain why it is easy to demonstrate the superiority of Sig/E7/LAMP-1 over E7 in the antitumor effect if it is administered in the form of recombinant vaccinia, but not if it is administered in the form of naked DNA vaccine.

In this study, we have abided by a 30-day time-frame for in vivo tumor protection and treatment experiments in the lung/liver metastasis model. Similarly to a previous study, ${ }^{8}$ we have used this time-frame to obtain results since information about metastatic tumors is unobtainable from the exterior, requiring mice to be killed at a given deadline. In the future, it would be interesting to expand the 30-day time-frame in order to determine the length of protective immunity generated by E7-specific DNA vaccines. Furthermore, in order to determine if repeated DNA vaccination is able to control established tumor nodules, we would first determine if a higher dosage of TC- 1 or increased time between tumor challenge and initial vaccination would generate established tumor nodules before DNA adminstration. We could then proceed to use different regimens of DNA vaccination, varying the booster dosage or the booster frequency in order to identify the optimal regimen for DNA administration. The information generated would provide helpful insights into the long-term control of tumor growth by DNA vaccines.

In summary, our results indicate that the E7-expressing DNA vaccine is an effective vaccine for preventing and treating HPV-16 E7-expressing tumor metastases in the liver or lungs. Both E7 DNA and Sig/E7/LAMP-1 DNA

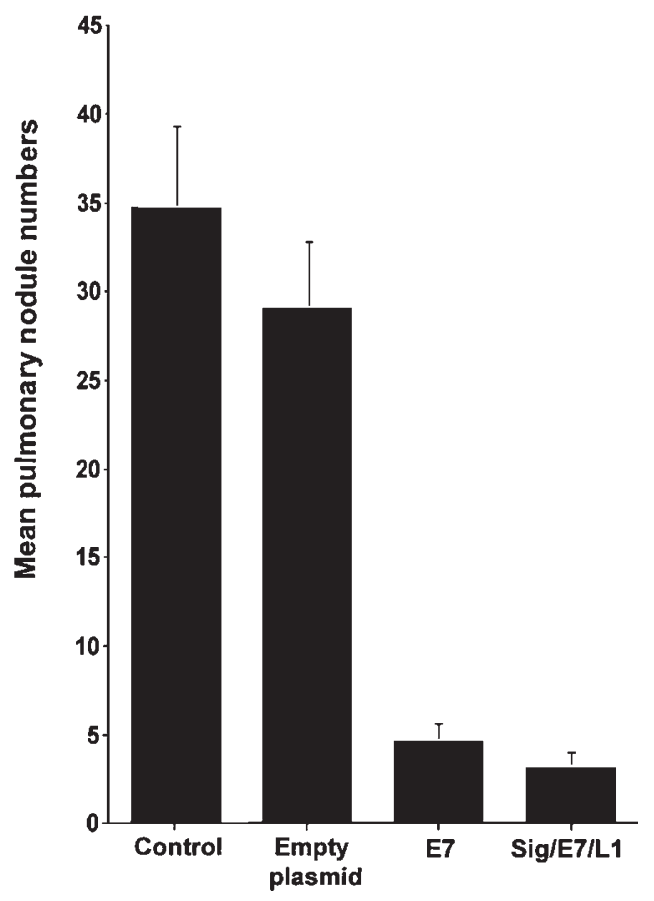

Figure 10 E7-expressing DNA vaccines eradicate previously established TC-1 tumors in the lungs. C57BL/6 mice (10 per group) were challenged with TC-1 tumor cells via intravenous tail vein injection at a dose of $5 \times 10^{4}$ cells per mouse. One week after the tumor challenge, mice were immunized with empty plasmid, E7 DNA or Sig/E7/LAMP-1 DNA via gene gun. Mice were monitored twice a week and were killed at day 30 after tumor challenge. The mean pulmonary nodule numbers ( \pm s.e.) shown here are from one representative experiment of three performed.

have potent antitumor effects, however, Sig/E7/LAMP1 DNA generates more potent tumor protection than E7 DNA when tested under more stringent conditions. In addition, Sig/E7/LAMP-1 DNA generates the strongest E7-specific CTL activity, the highest numbers of E7-specific $\mathrm{CD}^{+} \mathrm{T}$ cell precursors and the highest titer of antiE7 antibody. Although our tumor metastasis models were designed for HPV-16 E7 antigen-containing tumors, similar strategies can potentially be applied to other cancer systems with known tumor-specific antigens. As more tumor-specific antigens are characterized, using recent advances in molecular immunology, DNA vaccination will become more widely applicable for the treatment of cancer metastases.

\section{Materials and methods}

\section{Plasmid DNA constructs and preparation}

The Sig/E7/LAMP-1 chimeric gene was generated as described earlier. ${ }^{33}$ The chimeric gene and the HPV-16 E7 gene were cloned into the unique BamHI and EcoRI cloning sites of the $\mathrm{pCMV}$ neo expression vector downstream of the cytomegalovirus promoter. ${ }^{33}$ The schematic diagrams of the wild-type E7 and Sig/E7/LAMP-1 constructs are shown in Figure 1. The plasmid DNAs were transfected into subcloning efficient $\mathrm{DH} 5 \alpha^{\mathrm{TM}}$ cells (Life Technologies, Rockville, MD, USA). DNAs were then amplified and purified by double $\mathrm{CsCl}$ purification (BioServe Biotechnologies, Laurel, MD, USA). The integrity of plasmid DNA and the absence of Escherichia coli 


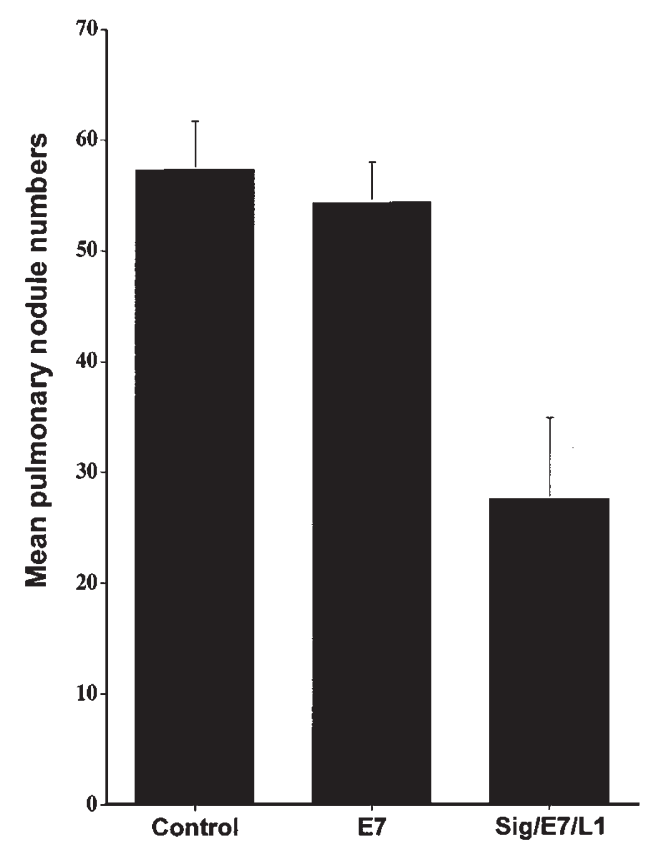

Figure 11 Sig/E7/LAMP-1 DNA generates more potent protective effect against the growth of TC-1 tumors in the lungs. C57BL/6 mice (10 per group) were immunized with E7 DNA or Sig/E7/LAMP-1 DNA via gene gun. In contrast to the previous set of experiments, the mice were vaccinated once without further booster. One week after the vaccination, mice were challenged with TC-1 tumor cells via intravenous tail vein injection at a dose of $1 \times 10^{5}$ cells per mouse. Mice were monitored twice a week and were killed at day 21 after tumor challenge. Mice vaccinated with Sig/E7/LAMP-1 DNA had significantly lower mean pulmonary nodule numbers than mice vaccinated with E7 DNA.

DNA or RNA were checked in each preparation using $1 \%$ agarose gel electrophoresis. DNA concentration was determined by the optical density measured at $260 \mathrm{~nm}$. The presence of inserted Sig/E7/LAMP-1 and E7 fragments was confirmed by restriction enzyme digestion and gel electrophoresis.

\section{DNA vaccination}

Gene gun particle-mediated DNA vaccination was done using a helium-driven gene gun (Bio-Rad, Hercules, CA, USA) according to the protocol provided by the manufacturer. Briefly, DNA-coated gold particles were prepared by combining $25 \mathrm{mg}$ of $1.6 \mu \mathrm{m}$ gold microcarriers (Biorad) and $100 \mu \mathrm{l}$ of $0.05 \mathrm{M}$ spermidine (Sigma, St Louis, $\mathrm{MO}$, USA). Plasmid DNA $(50 \mu \mathrm{g})$ and $1.0 \mathrm{M} \mathrm{CaCl}_{2}$ $(100 \mu \mathrm{l})$ were added sequentially to the microcariers while mixing by vortex. This mixture was allowed to precipitate at room temperature for $10 \mathrm{~min}$. The microcarrier/DNA suspension was then centrifuged (16000 $g$ for $5 \mathrm{~s}$ ) and washed three times in fresh absolute ethanol before resuspending in $3 \mathrm{ml}$ of polyvinylpyrrolidone $(0.1 \mathrm{mg} / \mathrm{ml})$ (Bio-rad) in absolute ethanol. The solution was then loaded into tubing and allowed to settle for $4 \mathrm{~min}$. The ethanol was gently removed and the microcarrier/DNA suspension was evenly attached to the inside surface of the tubing by rotating the tube. The tube was then dried by 0.4 liter per minute flowing nitrogen gas. The dried tubing coated with microcarrier/DNA was then cut to 0.5-inch cartridges and stored in a capped dry bottle at $4^{\circ} \mathrm{C}$. As a result, each cartridge contained
$1 \mu \mathrm{g}$ of plasmid DNA and $0.5 \mathrm{mg}$ of gold. The DNAcoated gold particles ( $1 \mu \mathrm{g}$ DNA per bullet) were delivered to the shaved abdominal region of the mice using a helium-driven gene gun (Bio-rad) with a discharge pressure of 400 p.s.i.

\section{ELISA}

The anti-HPV-16 E7 antibodies in the sera were determined by a direct ELISA as previously described. ${ }^{33}$ A 96microwell plate was coated with $100 \mu \mathrm{l}$ of $10 \mu \mathrm{g} / \mathrm{ml}$ bacteria-derived HPV-16 E7 protein at $4^{\circ} \mathrm{C}$ overnight and then the wells were blocked with phosphate-buffered saline (PBS) containing 20\% fetal bovine serum. Sera were prepared from the mice on day 14 after immunization, serially diluted in PBS, added to the ELISA wells, and incubated at $37^{\circ} \mathrm{C}$ for $2 \mathrm{~h}$. After washing with PBS containing $0.05 \%$ Tween-20, the plate was incubated with $1 / 2000$ dilution of a peroxidase-conjugated rabbit antimouse IgG antibody (Zymed, San Francisco, CA, USA) at room temperature for $1 \mathrm{~h}$. The plate was washed six times, developed with TMB (Pierce, Rockford, IL, USA), and stopped with $1 \mathrm{M} \mathrm{H}_{2} \mathrm{SO}_{4}$. The ELISA plate was read with a standard ELISA reader at $450 \mathrm{~nm}$.

\section{CTL assay}

CTL assay was done in a standard ${ }^{51} \mathrm{Cr}$ release assay as described before. ${ }^{33}$ Briefly, mouse spleen cells from each group were harvested at day 14 after vaccination. The splenocytes were cultured for 6 days in the presence of $1 \mu \mathrm{g} / \mathrm{ml}$ E7-specific H-2D ${ }^{\mathrm{b}}$ CTL epitope (E7 49-57, RAHYNIVTF) ${ }^{40}$ and 20 units $/ \mathrm{ml}$ murine interleukin-2. A standard $4 \mathrm{~h}$ chromium release assay was performed against MC57G ( $\mathrm{H}-2^{\mathrm{b}}$ ) cells pulsed with E7 peptide (aa 49-57) and labeled with $0.2 \mathrm{mCi}$ of $\mathrm{Na}_{2}{ }^{51} \mathrm{CrO}_{4}$ (Amersham, Piscataway, NJ, USA). The mean percent specific lysis of triplicate wells was calculated as: specific lysis $=[$ (c.p.m. experimental release - c.p.m. spontaneous release)/(c.p.m. maximum release - c.p.m. spontaneous release) $\times 100$.

\section{ELISPOT assay}

The ELISPOT assay described by Miyahira et $a l^{41}$ and Murali-Krishna et al ${ }^{13}$ was modified to detect HPV-16 E7specific $\mathrm{CD}^{+} \mathrm{T}$ cells. The ELISPOT assay was used to determine the number of antigen-specific $\mathrm{T}$ cell precursors based on the secretion of IFN- $\gamma$ by individual activated $\mathrm{T}$ cells. Therefore, it was important to incubate the plates with anti-mouse IFN- $\gamma$ antibody in order to capture the IFN- $\gamma$ secretion by these activated T cells. The 96well filtration plates (Millipore, Bedford, MA, USA) were coated with $10 \mu \mathrm{g} / \mathrm{ml}$ rat anti-mouse IFN- $\gamma$ antibody (clone R4-6A2; Pharmingen, San Diego, CA, USA) in $50 \mu \mathrm{l}$ of PBS. After overnight incubation at $4^{\circ} \mathrm{C}$, the wells were washed and blocked with culture medium containing $10 \%$ fetal bovine serum. Serial dilutions of fresh isolated spleen cells from each vaccinated mouse group, starting from $1 \times 10^{6}$ per well, were added to the well along with $15 \mathrm{U} / \mathrm{ml}$ interleukin-2. Cells were incubated at $37^{\circ} \mathrm{C}$ for $24 \mathrm{~h}$ either with or without $1 \mu \mathrm{g} / \mathrm{ml} \mathrm{E7-spe-}$ cific H-2D ${ }^{\mathrm{b}}$ CTL epitope (E7, aa 49-57). After culture, the plate was washed and followed by incubation with $5 \mu \mathrm{g} / \mathrm{ml}$ biotinylated IFN- $\gamma$ antibody (clone XMG1.2; Pharmingen) in $50 \mu \mathrm{l}$ PBS at $4^{\circ} \mathrm{C}$ overnight. After six washes, $1.25 \mu \mathrm{g} / \mathrm{ml}$ avidin-alkaline phosphatase (Sigma, St Louis, MO, USA) in $50 \mu$ l PBS was added and the plate 
was incubated for $2 \mathrm{~h}$ at room temperature. After washing, spots were developed by adding $50 \mu \mathrm{l} \mathrm{BCIP} / \mathrm{NBT}$ solution (Boehringer Mannheim, Indianapolis, IN, USA), and incubating at room temperature for $1 \mathrm{~h}$. The images of the spots in each well were captured by CCD video camera and the quantification of spot numbers was performed on a Macintosh computer using the NIH Image program 1.6 (developed at the US National Institutes of Health and available on the Internet at http://rsb.info.nih.gov/nih-image/) according to the method used by McCutcheon et al..$^{42}$

\section{ELISA for cytokines}

Splenocytes $\left(4 \times 10^{6}\right)$ were harvested 2 weeks after the last vaccination and cultured with $10 \mu \mathrm{g} / \mathrm{ml}$ E7 protein in a total volume of $2 \mathrm{ml}$ of RPMI 1640, supplemented with $10 \%(\mathrm{v} / \mathrm{v})$ fetal bovine serum, 50 units $/ \mathrm{ml}$ penicillin/streptomycin, $2 \mathrm{mM}$ L-glutamine, $1 \mathrm{~mm}$ sodium pyruvate, $2 \mathrm{~mm}$ nonessential amino acids in a 24well tissue culture plate for $72 \mathrm{~h}$. The supernatants were harvested and assayed for the presence of IFN- $\gamma$ and IL4 using ELISA kits (Endogen, Woburn, MA, USA) according to the manufacturer's protocol.

\section{Murine tumor cell line}

The production and maintenance of TC- 1 cells has been described previously. ${ }^{7}$ Briefly, HPV-16 E6, E7 and ras oncogene were used to transform primary C57BL/6 mice lung epithelial cells. The cells were grown in RPMI 1640, supplemented with $10 \%(\mathrm{v} / \mathrm{v})$ fetal bovine serum, 50 units/ml penicillin/streptomycin, $2 \mathrm{~mm}$ L-glutamine, $1 \mathrm{~mm}$ sodium pyruvate, $2 \mathrm{~mm}$ nonessential amino acids and $0.4 \mathrm{mg} / \mathrm{ml} \mathrm{G} 418$ at $37^{\circ} \mathrm{C}$ with $5 \% \mathrm{CO}_{2}$. On the day of tumor challenge, TC-1 cells were harvested by trypsinization, washed twice with $1 \times$ Hank's buffered salt solution and finally resuspended in $1 \times$ Hank's buffered salt solution to the designated concentration for injection.

\section{Mice and tumor cell inoculation}

Mice used in this study were purchased as 6- to 8-week old male C56BL/5 mice from the National Cancer Institute (Frederick, MD, USA) and kept in the oncology animal facility of the Johns Hopkins Hospital (Baltimore, MD, USA). All animal procedures were performed according to approved protocols and in accordance with recommendations for the proper use and care of laboratory animals. For the hepatic metastasis model, the mice were anesthetized with intraperitoneal injection of $0.1 \mathrm{ml}$ solution containing ketamine hydrochloride $(25 \mathrm{mg} / \mathrm{ml})$, xylazine $(2.5 \mathrm{mg} / \mathrm{ml})$, and $14.25 \%$ ethyl alcohol diluted $1: 3$ in $0.9 \% \mathrm{NaCl}$ solution. A small midline laparotomy incision was made and the left lobe of the liver was exteriorized. TC-1 cells in $10 \mu$ l Hank's buffered salt solution were injected with a 30-gauge needle directly into the liver parenchyma. The localized injection was confirmed by blanching of the liver parenchyma. Gentle compression for $60 \mathrm{~s}$ was used to minimize cellular extravasation and promote hemostasis. The liver was then placed back into the peritoneal cavity, and the incision was closed with metal wound clips. For the lung metastasis model, the TC- 1 cells in $100 \mu \mathrm{l}$ HBSS were injected into each mouse's tail vein.

\section{In vivo tumor protection experiments}

For the tumor protection experiment, mice were vaccinated via gene gun with $2 \mu \mathrm{g}$ of either Sig/E7/LAMP-1
DNA, E7 DNA, or the control plasmid without insert. One week later, the mice were boosted with the same regimen as in the first vaccination. In total, each mouse received $4 \mu \mathrm{g}$ of plasmid DNA. One week after the second vaccination, mice were challenged with TC-1 tumor cells at a dose of $1 \times 10^{4}$ cells per mouse for the hepatic metastasis model (five mice per group) and $5 \times 10^{4}$ TC- 1 cells per mouse for the lung metastasis model (10 mice per group). Mice were monitored twice a week and were killed at day 30 after tumor challenge.

\section{In vivo tumor regression experiments}

Tumor cells for injection and the DNA vaccines for immunization were prepared as described above. The mice were challenged with TC- 1 tumor cells first: $1 \times 10^{4}$ cells per mouse for the hepatic metastasis model (five mice per group), and $5 \times 10^{4} \mathrm{TC}-1$ cells per mouse for the lung metastasis model (10 mice per group for both tumor doses). Seven days after challenge with TC-1 tumor cells, mice were given $2 \mu \mathrm{g}$ of Sig/E7/LAMP-1 DNA, E7 DNA or control plasmid via gene gun. One week later, these mice were boosted with the same regimen as the first vaccination. In total, each mouse received $4 \mu \mathrm{g}$ of plasmid DNA. Mice were monitored twice a week and were killed at day 30 after tumor challenge.

\section{Tumor assessment and histologic studies}

For the hepatic metastasis model, the livers were removed after killing the mice and were examined in the fresh state by inspection and palpation for the presence of tumor. The tumor size was measured in animals in which tumor was found. For the lung metastasis model, the lungs were removed after killing the mice. The pulmonary metastasis nodules were counted under dissecting microscope. All hepatic specimens and those pulmonary specimens without gross visible tumors were then fixed with $10 \%$ neutral buffered formalin, embedded in paraffin and cut into $5 \mu \mathrm{m}$ sections from different levels. After hematoxylin and eosin staining, the slides were examined using a conventional microscope.

\section{Statistical analysis}

Student's $t$ test was used to compare the ELISPOT numbers and mean pulmonary nodules among different groups. $P$ values (two-tailed) less than 0.05 were considered statistically significant.

\section{Acknowledgements}

We would like to thank Drs Tian-Li Wang, Chien-Fu Hung and Robert J Kurman for helpful discussions. We would also like to thank Haiyan Chen for superb technical help and Morris Ling for preparation of the manuscript. This work was supported by NIH 5 po1 34582-01, U19 CA72108-02, RO1 CA72631-01 and the Richard W TeLinde endowment.

\section{References}

1 Chen $\mathrm{CH}, \mathrm{Wu}$ TC. Experimental vaccine strategies for cancer immunotherapy. J Biomed Sci 1998; 5: 231-252.

2 Sznol M, Holmlund J. Antigen-specific agents in develoment (review). Semin Oncol 1997; 24: 173-186.

3 Jaffee EM, Pardoll DM. Considerations for the clinical development of cytokine gene-transduced tumor cell vaccines. Methods 1997; 12: 143-153. 
4 Pardoll DM, Beckerleg AM. Exposing the immunology of naked DNA vaccines (review). Immunity 1995; 3: 165-169.

5 Donnelly JJ, Ulmer JB, Liu MA. DNA vaccines (review). Life Sci 1997; 60: 163-172.

6 Robinson HL, Torres CA. DNA vaccines. Semin Immunol 1997; 9: 271-283.

7 Lin K-Y et al. Treatment of established tumors with a novel vaccine that enhances major histocompatibility class II presentation of tumor antigen. Cancer Res 1996; 56: 21-26.

$8 \mathrm{Ji} \mathrm{H}$ et al. Antigen-specific immunotherapy for murine lung metastatic tumors expressing human papillomavirus type $16 \mathrm{E} 7$ oncoprotein. Int J Cancer 1998; 78: 41-45.

9 Hanke $\mathrm{T}$ et al. Enhancement of MHC class-I-restricted peptidespecific $\mathrm{T}$ cell induction by a DNA prime/MVA boost vaccination regime. Vaccine 1998; 16: 439-445.

10 Henriksen E. The lymphatic spread of carcinoma of the cervix and of the body of the uterus: a study of 420 necropsies. Am J Obstet Gynecol 1949; 58: 924-942.

11 Gilboa E. Murine models for cancer immunotherapy using cytokine gene modified tumor vaccines. In: Forni G, Foa R, Santoni A, Frati L (eds). Cytokine-induced Tumor Immunogeneity. Academic Press: London, 1994, pp 130-143.

12 Melief CJ, Kast WM. T-cell immunotherapy of tumors by adoptive transfer of cytotoxic $\mathrm{T}$ lymphocytes and by vaccination with minimal essential epitopes. Immunol Rev 1995; 145: 167-177.

13 Murali-Krishna K et al. Counting antigen-specific CD8 T cells: a reevaluation of bystander activation during viral infection. Immunity 1998; 8: 177-187.

14 Conry RM et al. A carcinoembryonic antigen polynucleotide vaccine has in vivo antitumor activity. Gene Therapy 1995; 2 $59-65$.

15 Syrengelas AD, Chen TT, Levy R. DNA immunization induces protective immunity against B-cell lymphoma. Nature Med 1996; 2: 1038-1041.

16 Bright RK et al. Protection against a lethal challenge with SV40transformed cells by the direct injection of DNA-encoding SV40 large tumor antigen. Cancer Res 1996; 56: 1126-1130.

17 Schirmbeck R, Bohm W, Reimann J. DNA vaccination primes MHC class I-restricted, simian virus 40 large tumor antigen-specific CTL in $\mathrm{H}-2 \mathrm{~d}$ mice that reject syngeneic tumors. J Immunol 1996; 157: 3550-3558.

18 Rosato A et al. CTL response and protection against P815 tumor challenge in mice immunized with DNA expressing the tumorspecific antigen P815A. Hum Gene Ther 1997; 8: 1451-1458.

19 Geissler $M$ et al. Genetic immunization with the free human chorionic gonadotropin beta subunit elicits cytotoxic T lymphocyte responses and protects against tumor formation in mice. Lab Invest 1997; 76: 859-871.

20 Schreurs MW et al. Genetic vaccination against the melanocyte lineage-specific antigen gp100 induces cytotoxic T lyphocytemediated tumor protection. Cancer Res 1998; 58: 2509-2514.

21 Chen $\mathrm{Y}$ et al. DNA vaccines encoding full-length or truncated Neu induce protective immunity against Neu-expressing mammary tumors. Cancer Res 1998; 58: 1965-1971.

22 Iwasaki A, Barber BH. Induction by DNA immunization of a protective antitumor cytotoxic $\mathrm{T}$ lymphocyte response against a minimal-epitope-expressing tumor. Cancer Immunol Immunother 1998; 45: 273-279.

23 Ciernik IF, Berzofsky JA, Carbone DP. Induction of cytotoxic T lymphocytes and antitumor immunity with DNA vaccines expressing single T cell epitopes. J Immunol 1996; 156: 2369-2375.
24 Irvine KR et al. Cytokine enhancement of DNA immunization leads to effective treatment of established pulmonary metastases. J Immunol 1996; 156: 238-245.

25 Ross HM et al. Priming for T-cell-mediated rejection of established tumors by cutaneous DNA immunization. Clin Cancer Res 1997; 3: 2191-2196.

26 Ulmer JB et al. Heterologous protection against influenza by injection of DNA encoding a viral protein. Science 1993; 259: 1745-1749.

27 Sparwasser T et al. Bacterial DNA and immunostimulatory CpG oligonucleotides trigger maturation and activation of murine dendritic cells. Eur J Immunol 1998; 28: 2045-2054.

28 Cohen AD, Boyer JD, Weiner DB. Modulating the immune response to genetic immunization. FASEB J 1998; 12: 1611-1626.

29 Condon $\mathrm{C}$ et al. DNA-based immunization by in vivo transfection of dendritic cells. Nature Med 1996; 2: 1122-1128.

30 Iwasaki A et al. The dominant role of bone marrow-derived cells in CTL induction following plasmid DNA immunization at different sites. J Immunol 1997; 159: 11-14.

31 Porgador A et al. Predominant role for directly transfected dendritic cells in antigen presentation to CD8+ cells after gene gun immunization. I Exp Med 1998; 188: 1075-1082.

32 Klinman DM et al. Contribution of cells at the site of DNA vaccination to the generation of antigen-specific immunity and memory. J Immunol 1998; 160: 2388-2392.

$33 \mathrm{Wu}$ TC et al. Engineering an intracellular pathway for major histocompatibility complex class II presentation of antigens. Proc Natl Acad Sci USA 1995; 92: 11671-11675.

34 Ruff AL et al. The enhanced immune response to the HIV gp160/LAMP chimeric gene product targeted to the lysosome membrane protein trafficking pathway. J Biol Chem 1997; 272: 8671-8678.

35 Boyle JS, Koniaras C, Lew AM. Influence of cellular location of expressed antigen on the efficacy of DNA vaccination: cytotoxic $\mathrm{T}$ lymphocyte and antibody responses are suboptimal when antigen is cytoplasmic after intramuscular DNA immunization. Int Immunol 1997; 9: 1897-1906.

36 Choi $\mathrm{AH}$ et al. Particle-bombardment-mediated DNA vaccination with rotavirus VP4 or VP7 induces high levels of serum rotavirus IgG but fails to protect mice against challenge. Virology 1998; 250: 230-240.

37 Akbari $\mathrm{O}$ et al. DNA vaccination: transfection and activation of dendritic cells as key events for immunity. J Exp Med 1999; 189: 169-178.

38 Sigal LJ et al. Cytotoxic T-cell immunity to virus-infected nonhaematopoietic cells requires presentation of exogenous antigen. Nature 1999; 398: 78-80.

39 Bennett SR et al. Induction of a $\mathrm{CD}^{+}$cytotoxic $\mathrm{T}$ lymphocyte response by cross-priming requires cognate $\mathrm{CD}^{+} \mathrm{T}$ cell help. $J$ Exp Med 1997; 186: 65-70.

40 Feltkamp MC et al. Vaccination with cytotoxic T lymphcoyte epitope-containing peptide protects against a tumor induced by human papillomavirus type 16-transformed cells. Eur J Immunol 1993; 23: 2242-2249.

41 Miyahira Y et al. Quantification of antigen specific CD8+ T cells using an ELISPOT assay. J Immunol Meth 1995; 181: 45-54.

42 McCutcheon $\mathrm{M}$ et al. A sensitive ELISPOT assay to detect lowfrequency human T lymphocytes. J Immunol Meth 1997; 210: 149-166. 